\title{
Disfunción de prótesis mitral mecánica: ¿Intervención quirúrgica o tratamiento inmediato con fibrinolíticos?
}

\section{Resumen}

Existe en la actualidad controversia acerca del mejor tratamiento para los pacientes portadores de trombosis valvular protésica. Muchos factores deben ser tenidos en cuenta al momento de decidir entre la resolución quirúrgica o el tratamiento con fibrinolíticos sistémicos. Las guías americanas recomiendan en plano de igualdad ambas terapéuticas, poniendo énfasis en el análisis de cada caso en particular. Las guías europeas, en cambio, le dan mayor nivel de recomendación a la cirugía, dejando la fibrinólisis para aquellos pacientes con alto riesgo quirúrgico. Se presenta el caso de una paciente de 54 años, portadora de prótesis mitral mecánica implantada hace 15 años, disfuncionante, en clase funcional II de la New York Heart Association, y se discuten las opciones diagnósticas y terapéuticas.

Palabras clave: $\quad$ FALLA DE PRÓTESIS

PRÓTESIS VALVULARES CARDÍACAS

VÁLVULA MITRAL

CIRUGÍA CARDÍACA

FIBRINOLÍTICOS

\section{Mechanical mitral prosthesis dysfunction: Surgical intervention or immediate treatment} with fibrinolytics?

\section{Summary}

There is ongoing debate regarding the optimal treatment for patients with prosthetic valve thrombosis. Several issues must be taken into consideration before defining either surgical or fibrinolytic treatment. North American guidelines place both therapeutic modalities under the same recommendation grade with special emphasis on the analysis of each individual case. European guidelines place surgical treatment as the preferred option except in patients with high surgical risk. Thereby, we present the clinical case of a 54 year old female with dysfunctional mechanical mitral prosthesis implanted 15 years ago and functional class II of the New York Heart Association. Diagnostic and therapeutic approaches are discussed. Key words:

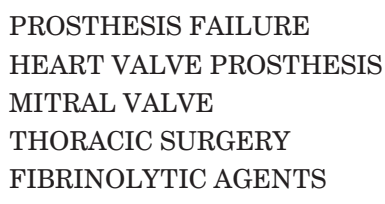

\section{Viñeta clínica}

Paciente de 54 años, sexo femenino, residente en Montevideo, portadora de fibrilación auricular (FA) permanente de varios años de evolución. Hace 15 años, sustitución valvular mitral por prótesis mecánica St. Jude de $27 \mathrm{~mm}$, por doble lesión de origen reumático. Tratada desde entonces con warfarina. Refiere controles frecuentes de INR aunque con valores muy inestables.

Hace un año presentó accidente cerebrovascular isquémico que evolucionó sin secuelas. El Doppler carotídeo no evidenció alteraciones y el ecocardiograma transtorácico (ETT) informó: prótesis mecánica mitral normofuncionante, sin masas evidentes; auriculomegalia izquierda, función sistólica del ventrículo izquierdo conservada.

Actualmente refiere historia de dos meses de evolución de disnea de esfuerzo clase funcional II, sin disnea de decúbito ni paroxística nocturna, por lo que consulta.

Al examen: normocoloreada, eupneica. Bien perfundida. Ritmo irregular con frecuencia cardíaca de $90 \mathrm{pm}$. Ruidos protésicos inaudibles en algunos latidos. Soplo diastólico de punta de intensidad variable. Presión arterial: 120/80 mmHg.

Estertores crepitantes bibasales.

INR actual: 2,4 .

Electrocardiograma: FA con frecuencia cardíaca media de $92 \mathrm{cpm}$. Hemibloqueo anterior izquierdo.

Radiografía de tórax: redistribución del flujo pulmonar, sin edema ostensible.

ETT: diámetros ventriculares izquierdos normales, con función sistólica conservada; aurícula izquierda: 50 $\mathrm{mm}$; prótesis mitral con gradiente medio de $20 \mathrm{mmHg}$. Impresiona hipomotilidad de uno de los discos, que se confirma mediante radioscopia.

Es ingresada en unidad cardiológica.

Ecocardiograma transesofágico (ETE): muestra un disco protésico fijo en posición cerrada con masa adyacente, inmóvil y de ecogenicidad intermedia. Mínimo reflujo mitral. Insuficiencia tricuspídea leve. Presión sistólica de arteria pulmonar: $50 \mathrm{mmHg}$. 


\section{La paciente debe ser intervenida quirúrgicamente The patient must receive surgical treatment Dr. Alberto Domenech}

\section{Análisis del caso clínico}

Se presenta una paciente de 54 años operada hace 15 años, a quien se le efectuó reemplazo valvular mitral por una prótesis mecánica de $27 \mathrm{~mm}$, con el antecedente de fibrilación auricular (FA) permanente $y$ un episodio de accidente cerebrovascular isquémico hace un año, actualmente sintomática por disnea en clase funcional (CF) II de dos meses de evolución. En la radiografía de tórax, si bien existe redistribución de flujo, se descarta edema agudo de pulmón. Tanto el ecocardiograma transtorácico como el transesofágico muestran con claridad trombosis protésica con inmovilidad de uno de los discos, con gradiente transmitral elevado y masa ocupante en ese sitio, aunque no se describe el tamaño de la misma.

\section{Discusión}

La trombosis valvular protésica (TVP) es hoy día una entidad poco frecuente, dado el estricto control de la anticoagulación oral (ACO) que sobre estos pacientes se realiza. Sin embargo, existen situaciones especiales que alteran esta rutina, principalmente enfermedades hepáticas en las que se altera el metabolismo de los anticoagulantes, interacciones medicamentosas o dietéticas, necesidad de conversión o suspensión de la ACO para procedimientos médicos invasivos, o simplemente un grupo de pacientes en los que la ACO es de muy difícil control para su estabilización.

La trombosis de una válvula protésica constituye una emergencia que al día de hoy puede ser tratada por vía quirúrgica a través de la sustitución de la válvula (SV) afectada, o por vía de la terapia fibrinolítica sistémica (TFS). Ambos procedimientos están avalados por las guías actuales de la American Heart Association/American College of Cardiology (ACC/AHA) en su modificación de $2017^{(1)}$ como recomendación de clase I y nivel de evidencia B-NR, tanto para la infusión lenta de bajas dosis de fibrinolíticos, como para el tratamiento quirúrgico de emergencia. Sin embargo, las guías de la Sociedad Europea de Cardiología y de la
Asociación Europea de Cirugía Cardio-torácica (ESC/EACTS) recomiendan la cirugía en casos graves sin comorbilidad seria (clase I, nivel C) y reserva la TFS para cuando la SV no está disponible o es de muy alto riesgo (clase IIa, nivel C) ${ }^{(2)}$.

Los datos actuales expresados en las guías muestran beneficio de la SV por sobre la TFS en:

1) Pacientes en CF IV.

2) Contraindicación para terapia fibrinolítica (cirugía reciente).

3) Trombo mayor a $0,8 \mathrm{~cm}^{2} \mathrm{o}$ trombosis recurrente.

4) Trombos en aurícula izquierda.

5) Presencia de pannus.

La mortalidad hospitalaria de la SV en pacientes de baja CF (I-II) es menor a 5\%, siendo la global, incluidos pacientes operados con edema agudo de pulmón, de alrededor de $10 \%$ a $15 \%(3,4)$.

En el caso de la TFS la mortalidad a 30 días reportada es de 7\%, pero con una efectividad solo de $75 \%$, y con $13 \%$ de eventos tromboembólicos, $6 \%$ de sangrado mayor, incluyendo $3 \%$ de hemorragias cerebrales ${ }^{(5)}$. Publicaciones más recientes muestran 90\% de éxito con la TFS, con menor tasa de eventos tromboembólicos y de sangrado mayor ${ }^{(6)}$.

Si bien no hay datos de series importantes en cuanto al número de pacientes, tanto los trabajos retrospectivos como metaanálisis y guías, recomiendan el análisis de cada caso en particular para elegir el mejor tratamiento.

Karthikeyan publicó en 2015 un metaanálisis sobre 690 pacientes con trombosis protésica. Si bien no encontró diferencias significativas de mortalidad a 30 días, sí las hubo a favor de la SV en eventos tromboembólicos, sangrado mayor y trombosis protésica recurrente ${ }^{(7)}$.

En el caso que nos ocupa, si bien no se informa el tamaño del trombo, sí se asegura la inmovilidad de un disco en posición cerrada. Además, no podemos descartar la presencia de pannus en la prótesis, teniendo en cuenta que la misma lleva 15 años de implantada.

Servicio de Cirugía Cardiovascular, Hospital Italiano de Buenos Aires, Argentina.

Correspondencia: Dr. Alberto Domenech. Correo electrónico: alberto.domenech@hospitalitaliano.org.ar

$\mathrm{El}$ autor declara no tener conflictos de intereses.

Recibido Ago 31, aceptado Set 12, 2018. 
Por otra parte, la paciente se encuentra en buen estado general, eupneica y bien perfundida, reuniendo así las condiciones en las que la cirugía ha mostrado mejores resultados.

Otro punto a considerar a favor de la SV es la dificultad para poder mantener en el tiempo una ACO estable y suficiente en esta paciente. Esto permitiría considerar la posibilidad de realizar la SV por una válvula biológica con tratamiento anticalcificante. Si bien la paciente requerirá ACO definitiva por su auriculomegalia izquierda y FA permanente, la posibilidad de ACO insuficiente no influirá sobre el normal funcionamiento de la válvula sustituida.

\section{Bibliografía}

1. Nishimura R, Otto C, Bonow R, Carabello B, Erwin J3rd, Fleisher L, et al. 2017 AHA/ACC focused update of the 2014 AHA/ACC guideline for the management of patients with valvular heart disease: a report of the American College of Cardiology/American Heart Association task force on clinical practice guidelines. Circulation 2017; 135(25):e1159-95.

2. Baumgartner H, Falk V, Bax J, De Bonis M, Hamm C, Holm P, et al. 2017 ESC/EACTS Guidelines for the management of valvular heart disease. Eur Heart J 2017; 38(36):2739-91.
3. Keuleers S, Herijgers P, Herregods M, Budts W, Dubois C, Meuris B, et al. Comparison of thrombolysis versus surgery as a first line therapy for prosthetic heart valve thrombosis. Am J Cardiol 2011; 107(2):275-9.

4. Roudaut R, Lafitte S, Roudaut M, Reant P, Pillois X, Durrieu-Jaïs C, et al. Management of prosthetic heart valve obstruction: fibrinolysis versus surgery. Early results and long-term follow-up in a single-centre study of 263 cases. Arch Cardiovasc Dis $2009 ; 102(4): 269-77$.

5. Nagy A, Dénes M, Lengyel M. Predictors of the outcome of thrombolytic therapy in prosthetic mitral valve thrombosis: a study of 62 events. J Heart Valve Dis $2009 ; 18(3): 268-75$.

6. Özkan M, Gündüz S, Gürsoy $\mathbf{O}$, Karakoyun $\mathbf{S}$, Astarcýoðlu M, Kalçýk M, et al. Ultraslow thrombolytic therapy: a novel strategy in the management of PROsthetic MEchanical valve Thrombosis and the prEdictors of outcomE: The Ultra-slow PROMETEE trial. Am Heart J 2015; 170(2):409-18.

7. Karthikeyan G, Senguttuvan N, Joseph J, Devasenapathy N, Bahl V, Airan B. Urgent surgery compared with fibrinolytic therapy for the treatment of left-sided prosthetic heart valve thrombosis: a systematic review and meta-analysis of observational studies. Eur Heart J 2013; 34(21):1557-66.

\section{La paciente requiere tratamiento inmediato con fibrinolíticos The patient requires immediate treatment with fibrinolytics \\ Dr. Daniel José Piñeiro}

\section{Análisis del caso clínico}

Los datos de mayor relevancia en este caso son:

- Enfermedad actual: disnea clase funcional II de dos meses de evolución.

- Antecedentes: reemplazo de válvula mitral por prótesis mecánica. Fibrilación auricular crónica. Accidente cerebrovascular isquémico de probable mecanismo embólico atribuible a la fibrilación auricular (ausencia de patología carotídea o disfunción protésica [valorada solo por eco Doppler transtorácico]). Anticoagula- ción oral con antagonista de la vitamina $\mathrm{K}$ con controles "inestables".

\section{Exámenes complementarios}

- Ecocardiografía: gradiente medio transprotésico $20 \mathrm{mmHg}$. Un disco protésico fijo con masa adyacente de ecogenicidad intermedia e inmóvil (confirmado por radioscopia). Presión sistólica de la arteria pulmonar $50 \mathrm{mmHg}$.

Hospital de Clínicas José de San Martín, Universidad de Buenos Aires, Argentina

Correspondencia: Dr. Daniel José Piñeiro. Correo electrónico: djpineiro@gmail.com

El autor declara no tener conflictos de intereses.

Recibido Set 3, 2018; aceptado Set 12, 2018 


\begin{tabular}{|c|c|c|c|c|c|}
\hline & & & Normal & $\begin{array}{l}\text { Obstrucción } \\
\text { posible }\end{array}$ & $\begin{array}{l}\text { Obstrucción } \\
\text { significativa }\end{array}$ \\
\hline Cualitativo & \multicolumn{2}{|c|}{ Estructura y motilidad valvular } & Normal & $\begin{array}{c}\text { Frecuentemente } \\
\text { anormal }\end{array}$ & Anormal \\
\hline Semicuantitativo & \multicolumn{2}{|c|}{ Tiempo de hemipresión (ms) } & $<130$ & $130-200$ & $>200$ \\
\hline \multirow[t]{8}{*}{ Cuantitativo } & \multirow[t]{4}{*}{ Dependientes del flujo } & Velocidad pico $(\mathrm{m} / \mathrm{s})$ & $<1,9$ & $1,9-2,5$ & $>2,5$ \\
\hline & & Gradiente medio (mm Hg) & $<6$ & $6-10$ & $>10$ \\
\hline & & $\begin{array}{l}\text { Aumento del gradiente medio } \\
\text { durante el eco-estrés) }\end{array}$ & $<5$ & $5-12$ & $>12$ \\
\hline & & $\begin{array}{l}\text { Aumento del gradiente medio } \\
\text { en el seguimiento }\end{array}$ & $<3$ & $3-5$ & $>5$ \\
\hline & \multirow[t]{3}{*}{ Independientes del flujo } & ORE $\left(\mathrm{cm}^{2}\right)$ & $>2$ & $1-2$ & $<1$ \\
\hline & & $\begin{array}{l}\text { ORE vs valores de referencia } \\
\text { normales }\end{array}$ & $\begin{array}{l}\text { Referencia } \\
\quad \pm 1 \mathrm{DS}\end{array}$ & $\begin{array}{l}<\text { Referencia } \\
\quad-\text { 1DS }\end{array}$ & $\begin{array}{c}<\text { Referencia } \\
- \text { 2DS }\end{array}$ \\
\hline & & $\begin{array}{l}\text { Diferencia (ORE de referencia } \\
\text { - ORE medido })\left(\mathrm{cm}^{2}\right)\end{array}$ & $<0,25$ & $0,25-0,35$ & $>0,35$ \\
\hline & \multicolumn{2}{|c|}{ Indice de velocidad Doppler } & $<2,2$ & $2,2-2,5$ & $>2,5$ \\
\hline
\end{tabular}

\section{Consideraciones diagnósticas}

\section{¿Cuándo sospechar clínicamente trombosis obstructiva de una prótesis mecánica en posición mitral?}

La tasa anual de trombosis en prótesis mecánicas en posición mitral es de 5/1.000 pacientes/año ${ }^{(1)}$. La trombosis protésica puede ser no obstructiva u obstructiva $^{(1)}$. En el primer caso su manifestación clínica más frecuente es el accidente cerebrovascular embólico, en el segundo caso su principal expresión es el compromiso hemodinámico que va desde la disnea progresiva al shock cardiogénico ${ }^{(1)}$. El examen clínico revela ausencia del "click" protésico de apertura y/o de cierre, nuevo soplo cardíaco, y signos de edema de pulmón e insuficiencia cardíaca ${ }^{(1)}$.

La historia clínica de la paciente que nos ocupa evidencia clínicamente la sospecha de trombosis obstructiva de la prótesis mecánica en posición mitral.

\section{¿Qué hacer ante la evidencia clínica de probable trombosis obstructiva de una prótesis mecánica en posición mitral?}

Los parámetros esenciales para la valoración de la función de una válvula protésica incluyen la información clínica, las imágenes (fundamentalmente la ecocardiografía), la evaluación por Doppler, los datos complementarios que brindan otras imágenes (cine- fluoroscopía, tomografía computada) y la comparación con estudios previos (si los hubiese y estuviesen disponibles) $)^{(2,3)}$. En el eco Doppler transtorácico son relevantes: la evaluación de los gradientes transvalvulares, la movilidad y el grosor de los discos y la presencia de masas, y en el eco Doppler transesofágico: las dimensiones del trombo ${ }^{(2,3)}$. En la tabla 1 se resumen los criterios para considerar obstructiva una trombosis de prótesis mecánica en posición mitral ${ }^{(3)}$. En estas circunstancias, debe realizarse siempre el diagnóstico diferencial entre trombosis y el desarrollo de una capa de tejido fibrovascular o de granulación denominada pannus ${ }^{(1-3)}$. En la tabla 2 se presentan los criterios diferenciales entre trombosis y pannus $^{(3)}$.

Las imágenes de la paciente evidencian clínicamente la probabilidad de trombosis obstructiva de la prótesis mecánica en posición mitral.

\section{Consideraciones terapéuticas}

\section{¿Qué hacer ante la evidencia clínica e imagenológica de trombosis obstructiva de una prótesis mecánica en posición mitral?}

La trombosis no obstructiva de una prótesis mecánica en posición mitral se observa, según algunos autores, en hasta $10 \%$ de los pacientes ${ }^{(1)}$. Se ha verificado que la optimización de la anticoagulación se asocia 


\begin{tabular}{|c|c|c|}
\hline & Pannus & Trombosis \\
\hline Cronología & $\begin{array}{l}\text { Mínimo } 12 \text { meses (comúnmente }>5 \\
\text { años desde la cirugía) }\end{array}$ & $\begin{array}{l}\text { Sucede en cualquier momento (si es } \\
\text { tardía suele asociarse a pannus) }\end{array}$ \\
\hline $\begin{array}{l}\text { Relación con la anticoagulación } \\
\text { (bajo INR) }\end{array}$ & $+/-$ & +++ \\
\hline Localización & Mitral > Aórtica & Tricúspide $>>$ Mitral = Aórtica \\
\hline \multirow[t]{5}{*}{ Morfología } & Masa pequeña & Masa mayor que el pannus \\
\hline & $\begin{array}{l}\text { Generalmente compromete la línea } \\
\text { de sutura (anillo) }\end{array}$ & $\begin{array}{l}\text { Comúnmente con motilidad } \\
\text { independiente }\end{array}$ \\
\hline & Crecimiento centrípeto & $\begin{array}{l}\text { Puede verse el anillo externo } \\
\text { delgado }\end{array}$ \\
\hline & Confinado al plano del disco & $\begin{array}{l}\text { En posición mitral, se proyecta hacia } \\
\text { la aurícula izquierda }\end{array}$ \\
\hline & Crecimiento por debajo del disco & Elementos móviles \\
\hline $\begin{array}{l}\text { Densidad ecocardiográfica (tasa de } \\
\text { video intensidad) }\end{array}$ & $\begin{array}{l}\text { Mayor ( }>0,7[100 \% \text { de } \\
\text { especificidad }])\end{array}$ & Menor $(<0,4)$ \\
\hline $\begin{array}{l}\text { Tomografía computada: valor de } \\
\text { atenuación }\end{array}$ & $>200 \mathrm{UH}$ & $<200 \mathrm{UH}$ \\
\hline Impacto en el gradiente & Aórtica > Mitral & Mitral > Aórtica \\
\hline Impacto en el ORE & Aórtica > Mitral & Mitral > Aórtica \\
\hline Impacto en la motilidad del disco & Sí /no & Sí \\
\hline
\end{tabular}

Tabla 3. Recomendaciones de la Sociedad Europea de Cardiología para el tratamiento de la trombosis de prótesis mecánica.

\begin{tabular}{lcc}
\hline Recomendación & Clase & Nivel de evidencia \\
\hline $\begin{array}{l}\text { Se recomienda el reemplazo valvular de urgencia o emergencia para la } \\
\text { trombosis obstructiva en pacientes críticos sin comorbilidades graves }\end{array}$ & $\mathrm{I}$ & $\mathrm{C}$ \\
$\begin{array}{l}\text { Debe considerarse la fibrinólisis (empleando activador tisular del } \\
\text { plasminógeno recombinante } 10 \mathrm{mg} \text { en bolo }+90 \mathrm{mg} \text { en } 90 \mathrm{~min} \text { con } \\
\text { heparina no fraccionada o estreptoquinasa } 1.500 .000 \text { U en } 60 \text { min sin } \\
\text { heparina no fraccionada) cuando no se disponga de cirugía o si el riesgo } \\
\text { quirúrgico es alto o si se trata de una prótesis derecha. }\end{array}$ & $\mathrm{IIa}$ & $\mathrm{C}$ \\
$\begin{array}{l}\text { Debe considerarse la cirugía para trombos no obstructivos grandes }(>10 \\
\text { mm) complicados con embolia }\end{array}$ & $\mathrm{IIa}$ & $\mathrm{C}$ \\
\hline
\end{tabular}

Reproducido de Baumgartner H, Falk V, Bax JJ, et al. 2017 ESC/EACTS Guidelines for the management of valvular heart disease. Eur Heart J. 2017;38:2739-91.

C: nivel de evidencia C, consenso de opinión de expertos o pequeños estudios, estudios retrospectivos, o registros.

con una mejor evolución y escasas complicaciones en pacientes con trombos pequeños $(<5 \mathrm{~mm})$. En pacientes con trombos mayores $(>5 \mathrm{~mm}$ ) aumenta el riesgo embólico(1)
En la paciente motivo del análisis, el antecedente de accidente cerebrovascular isquémico de probable mecanismo embólico fue atribuido a la fibrilación auricular. Sin embargo, la anticoagulación oral con antagonista de la vitamina $\mathrm{K}$ con controles 
Tabla 4. Recomendaciones de la Asociación Americana del Corazón y del Colegio Americano de Cardiología para el tratamiento de la trombosis de prótesis mecánica.

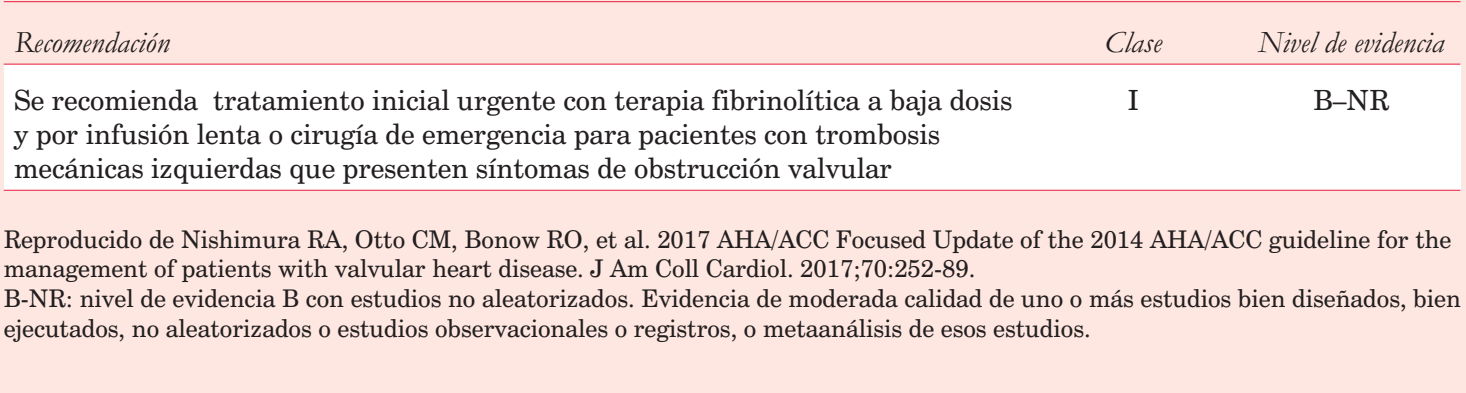

Tabla 5. Fibrinólisis versus cirugía para la trombosis protésica.

\begin{tabular}{|c|c|c|}
\hline & A favor de la cirugía & A favor de la fibrinólisis \\
\hline 1 & Disponibilidad inmediata de cirugía con experiencia & Ausencia de cirugía con experiencia \\
\hline 2 & Riesgo quirúrgico bajo & Riesgo quirúrgico alto \\
\hline 3 & Contraindicación de fibrinólisis & Ausencia de contraindicación de fibrinólisis \\
\hline 4 & Trombosis recurrente & Primer episodio de trombosis \\
\hline 5 & Clase funcional IV de la NYHA & Clase funcional I-III de la NYHA \\
\hline 6 & Trombo grande $\left(>0,8 \mathrm{~cm}^{2}\right)$ & Trombo pequeño $\left(\leq 0,8 \mathrm{~cm}^{2}\right)$ \\
\hline 7 & Trombo en la aurícula izquierda & Ausencia de trombo en la aurícula izquierda \\
\hline 8 & $\begin{array}{l}\text { Enfermedad coronaria concomitante que requiere } \\
\text { revascularización }\end{array}$ & $\begin{array}{l}\text { Ausencia de enfermedad coronaria o enfermedad } \\
\text { coronaria leve }\end{array}$ \\
\hline 9 & Enfermedad de otra válvula & Ausencia de enfermedad de otra válvula \\
\hline 10 & Posible pannus & Visualización del trombo \\
\hline 11 & Elección del paciente & Elección del paciente \\
\hline
\end{tabular}

“inestables", la ausencia de patología carotídea y la exclusión de trombosis protésica valorada solo por eco Doppler transtorácico, permiten especular que, si bien la presencia de fibrilación auricular es suficiente para explicar el evento, también sería posible que ya hubiera una trombosis protésica no obstructiva que no fue detectada mediante el eco Doppler transtorácico.

Más allá de esta hipótesis, la paciente presenta en el momento actual evidencias clínicas e imagenológicas de trombosis obstructiva de la prótesis mecánica en posición mitral. La conducta en esta circunstancia debe ser individualizada para cada paciente considerando los hallazgos imagenológicos, la factibilidad de realizar fibrinólisis y el riesgo de una eventual cirugía ${ }^{(1,4-9)}$. Las tablas 3 y 4 reprodu- cen las recomendaciones de las guías de la Sociedad Europea de Cardiología 2017 y de la Asociación Americana del Corazón y del Colegio Americano de Cardiología 2017 para el tratamiento de la trombosis de prótesis mecánica ${ }^{(10,11)}$. Estas últimas recomiendan terapia fibrinolítica a baja dosis y por infusión lenta o cirugía de emergencia, dependiendo de múltiples factores incluyendo la experiencia clínica y quirúrgica del equipo actuante ${ }^{(11)}$ (tabla 5). Si extrapolamos estos criterios a la paciente en análisis, se observa que favorece a la cirugía el riesgo quirúrgico que "prima facie" parece bajo (aunque se carece de datos para precisarlo). Por el contrario, favorece la fibrinólisis la ausencia "prima facie" de contraindicaciones para la misma, ser el primer episodio de trombosis, la clase funcional caracteri- 
zada como grado II, la aparente ausencia de enfermedad coronaria o de enfermedad de otra válvula y la aparente visualización de trombo en el ecocardiograma transesofágico. Cabe subrayar que se carece de información sobre la disponibilidad inmediata de cirugía con experiencia, el tamaño del trombo, la presencia o no de trombo en la aurícula izquierda y la posibilidad de pannus. Por último, pero no menos importante, se ignora la elección de la paciente.

\section{Conclusiones}

La conducta más apropiada para esta paciente debe ser individualizada y discutida por el heart team considerando también la elección de la paciente ante las opciones terapéuticas. En base a los criterios mencionados arriba, optaríamos por el tratamiento inmediato con fibrinolíticos dado que la paciente no presenta un estado crítico y ha estado inadecuadamente anticoagulada en el período reciente ${ }^{(10)}$.

\section{Bibliografía}

1. Lim W, Lloyd G, Bhattacharyya S. Mechanical and surgical bioprosthetic valve thrombosis. Heart 2017; 103(24):1934-41.

2. Zoghbi W, Chambers J, Dumesnil J, Foster E, Gottdiener J, Grayburn P, et al. Recommendations for evaluation of prosthetic valves with echocardiography and doppler ultrasound: a report From the American Society of Echocardiography's Guidelines and Standards Committee and the Task Force on Prosthetic Valves, developed in conjunction with the American College of Cardiology Cardiovascular Imaging Committee, Cardiac Imaging Committee of the American Heart Association, the European Association of Echocardiography, a registered branch of the European Society of Cardiology, the Japanese Society of Echocardiography and the Canadian Society of Echocardiography, endorsed by the American College of Cardiology Foundation, American Heart Association, European Association of Echocardiography, a registered branch of the European Society of Cardiology, the Japanese Society of Echocardiography, and Canadian Society of Echocardiography. J Am Soc Echocardiogr 2009; 22(9):975-1014.

3. Lancellotti P, Pibarot P, Chambers J, Edvardsen T, Delgado V, Dulgheru R, et al. Recommen- dations for the imaging assessment of prosthetic heart valves: a report from the European Association of Cardiovascular Imaging endorsed by the Chinese Society of Echocardiography, the Inter-American Society of Echocardiography, and the Brazilian Department of Cardiovascular Imaging. Eur Heart J Cardiovasc Imaging 2016; 17(6):589-90.

4. Dangas G, Weitz J, Giustino G, Makkar R, Mehran R. Prosthetic heart valve thrombosis. J Am Coll Cardiol 2016; 68(24):2670-89.

5. Huang G, Schaff H, Sundt T, Rahimtoola S. Treatment of obstructive thrombosed prosthetic heart valve. J Am Coll Cardiol 2013; 62(19):1731-6.

6. Karthikeyan G, Senguttuvan N, Joseph J, Devasenapathy N, Bahl V, Airan B. Urgent surgery compared with fibrinolytic therapy for the treatment of left-sided prosthetic heart valve thrombosis: a systematic review and meta-analysis of observational studies. Eur Heart J 2013; 34(21):1557-66.

7. Özkan M, Gündüz S, Biteker M, Astarcioglu M, Çevik C, Kaynak E, et al. Comparison of different TEE-guided thrombolytic regimens for prosthetic valve thrombosis: the TROIA trial. JACC Cardiovasc Imaging 2013; 6(2):206-16.

8. Özkan M, Gündüz S, Gürsoy $\mathbf{O}$, Karakoyun $\mathbf{S}$, Astarcýoðlu M, Kalçýk M, et al. Ultraslow thrombolytic therapy: a novel strategy in the management of PROsthetic MEchanical valve Thrombosis and the prEdictors of outcomE: the Ultra-slow PROMETEE trial. Am Heart J 2015; 170(2):409-18

9. Whitlock R, Sun J, Fremes S, Rubens F, Teoh K. Antithrombotic and thrombolytic therapy for valvular disease: Antithrombotic Therapy and Prevention of Thrombosis, 9th ed: American College of Chest Physicians Evidence-Based Clinical Practice Guidelines. Chest 2012; 141(2 Suppl):e576S-600S.

10. Baumgartner H, Falk V, Bax J, De Bonis M, Hamm C, Holm P, et al. 2017 ESC/EACTS Guidelines for the management of valvular heart disease. Eur Heart J 2017; 38(36):2739-91.

11. Nishimura R, Otto C, Bonow R, Carabello B, Erwin J3rd, Fleisher L, et al. 2017 AHA/ACC focused update of the 2014 AHA/ACC guideline for the management of patients with valvular heart disease: a report of the American College of Cardiology/American Heart Association task force on clinical practice guidelines. J Am Coll Cardiol 2017; 70(2):252-89. 


\section{Respuesta al Dr. Daniel José Piñeiro}

En base a lo expuesto en la minuta de historia clínica y los datos disponibles, reiteramos la opinión favorable a la cirugía, dado el aparente buen estado clínico general de la paciente y la ausencia de comorbilidades de importancia.

Como vemos, ambas posiciones de la controversia asientan fundamentalmente su indicación de tratamiento en este aspecto. Por ello, cito textual- mente la recomendación de las guías europeas: "Se recomienda el reemplazo valvular de urgencia o emergencia para la trombosis obstructiva en pacientes críticos sin comorbilidades graves", clase I, nivel de evidencia $\mathrm{C}$.

Asumimos, por cierto, que se cuenta con un equipo de cirugía cardiovascular con experiencia para resolver este caso.

Dr. Alberto Domenech

\section{Respuesta al Dr. Alberto Domenech}

Agradezco el privilegio de compartir esta controversia con mi amigo y reconocido cirujano cardiovascular, el Dr. Alberto Domenech. Coincido con él en que tanto la posibilidad de tratamiento médico como la de cirugía son apropiadas en este caso. Pienso que carecemos de información sobre algunos criterios que favorecerían la cirugía o la fibrinólisis expues- tos en la tabla 5. Reunidos estos datos, con la cuidadosa ponderación del heart team y la opinión informada de la paciente, se podrá tomar una decisión que integre la mejor evidencia disponible, la experiencia del grupo médico tratante y los valores de la paciente, honrando el paradigma actual de la medicina basada en la evidencia ${ }^{(1)}$.

Dr. Daniel José Piñeiro

\section{Bibliografía}

1. Djulbegovic B, Guyatt GH. Progress in evidence-based medicine: a quarter century on. Lancet 2017;390 (10092):415-23. doi: 10.1016/S0140-6736 (16)31592-6 